\title{
Assistência farmacêutica em idosos com tuberculose e a resistência ao tratamento
}

\author{
Pharmaceutical care for elderly people with tuberculosis and resistance to treatment \\ Atención farmacéutica a personas mayores con tuberculosis y resistencia al tratamento
}

Recebido: 19/11/2021 | Revisado: 24/11/2021 | Aceito: 03/12/2021 | Publicado: 09/12/2021

\author{
Woodle de Lima Pinto \\ ORCID: https://orcid.org/0000-0001-9395-7685 \\ Universidade Nilton Lins, Brasil \\ E-mail: woodleelima@gmail.com \\ Erick Frota Gomes Figueiredo \\ ORCID: https://orcid.org/0000-0002-6127-0544 \\ Universidade Nilton Lins, Brasil \\ E-mail: erick.figueiredo@uniniltonlins.edu.br
}

\begin{abstract}
Resumo
Os riscos em idosos com tuberculose são elevados, por isso a assistência farmacêutica nesse tipo de paciente é importante, sendo o farmacêutico o profissional mais capacitado para suporte de intervenções medicamentosas quando a resistência ao tratamento. O estudo teve como objetivo relacionar a atuação do profissional farmacêutico no suporte a idosos com resistência ao tratamento de tuberculose. O estudo trata-se de uma revisão de literatura, onde foi utilizado o método de pesquisa bibliográfica, pela possibilidade de analisar referências publicadas em estudos já realizados para o recolhimento amplo de informações e conhecimentos prévios acerca da temática. Para o levantamento do estudo foram utilizados Livros, Revistas, Diretrizes Sociedades Brasileiras, artigos nos bancos de pesquisas SciELO, PubMed/MEDLINE. Verificou-se que resistência ao tratamento é uma ameaça ao sistema de saúde, pois pode levar ao aumento do tempo de internação hospitalar, declínio funcional, aumento dos gastos com saúde e mortalidade por todas as causas. A eficácia do tratamento vai depender da disciplina e conhecimento do paciente sobre a utilização correta dos fármacos, além da compreensão dos benefícios que o tratamento é feito de forma como é prescrito e orientado pelo médico. Com isso, ter o farmacêutico na prática da assistência com o desenvolvimento de estratégias que possam promover a educação e sensibilização dos idosos em relação ao tratamento, é de grande importância para o controle e minimização das complicações da doença.
\end{abstract}

Palavras-chave: Assistência farmacêutica; Tuberculose; Resistência medicamentosa; Saúde do idoso.

\begin{abstract}
The risks in elderly people with tuberculosis are high, which is why pharmaceutical assistance in this type of patient is important, with the pharmacist being the most qualified professional to support drug interventions when there is resistance to treatment. The study aimed to relate the role of the pharmacist in supporting elderly people with resistance to tuberculosis treatment. The study is a literature review, where the bibliographic research method was used, due to the possibility of analyzing references published in studies already carried out for the collection of ample information and previous knowledge about the subject. For the study survey, Books, Magazines, Brazilian Societies Guidelines, articles in the SciELO, PubMed/MEDLINE research databases were used. Resistance to treatment was found to be a threat to the health system, as it can lead to increased length of hospital stay, functional decline, increased spending on health and mortality from all causes. The effectiveness of the treatment will depend on the patient's discipline and knowledge about the correct use of drugs, in addition to an understanding of the benefits that the treatment provided in the way it is prescribed and guided by the doctor. Thus, having the pharmacist in the care practice with the development of strategies that can promote the education and awareness of the elderly in relation to the treatment is of great importance for the control and minimization of the disease's complications.
\end{abstract}

Keywords: Pharmaceutical care; Tuberculosis; Drug resistance; Health of the elderly.

\section{Resumen}

Los riesgos en las personas mayores con tuberculosis son elevados, por lo que la asistencia farmacéutica en este tipo de pacientes es importante, siendo el farmacéutico el profesional más cualificado para apoyar las intervenciones farmacológicas cuando hay resistencia al tratamiento. El estudio tuvo como objetivo relacionar el papel del farmacéutico en el apoyo a las personas mayores con resistencia al tratamiento de la tuberculosis. El estudio es una revisión de la literatura, donde se utilizó el método de investigación bibliográfica, debido a la posibilidad de analizar referencias publicadas en estudios ya realizados para la recolección de amplia información y conocimientos previos sobre el tema. Para la encuesta de estudio se utilizaron libros, revistas, guías de sociedades brasileñas, artículos de las bases de datos de investigación SciELO, PubMed / MEDLINE. Se descubrió que la resistencia al tratamiento es una 
amenaza para el sistema de salud, ya que puede conducir a una mayor duración de la estancia hospitalaria, deterioro funcional, mayor gasto en salud y mortalidad por todas las causas. La efectividad del tratamiento dependerá de la disciplina y el conocimiento del paciente sobre el uso correcto de los medicamentos, además de la comprensión de los beneficios que brinda el tratamiento en la forma en que es prescrito y guiado por el médico. Así, contar con el farmacéutico en la práctica asistencial con el desarrollo de estrategias que puedan promover la educación y sensibilización de las personas mayores en relación al tratamiento es de gran importancia para el control y minimización de las complicaciones de la enfermedad.

Palabras clave: Atención farmacéutica; Tuberculosis; Resistencia a las drogas; Salud de los ancianos.

\section{Introdução}

Junto com o aumento do número da população idosa, tem ocorrido um aumento no número de casos de tuberculose entre os idosos. Embora essa doença seja evitável e curável, aproximadamente 200 casos de tuberculose são registrados por dia no Brasil, de acordo com dados do Ministérios da Saúde. Esse número ainda é considerado elevado, por se tratar de uma patologia que se for diagnosticada precocemente e completamente tratada, os pacientes rapidamente se tornam não infecciosos e, eventualmente, curados.

Como apresentado a prevalencia dessa doença na pesquisa realizada por Pereira et al. (2020) que buscou avaliar a incidência e indicadores do controle de Tuberculose, onde foram notificados mais de 88 casos de tuberculose em indivíduos com idade maior que 50 anos e em sua maioria homens. Costa et al. (2020) mostrou em seu estudo uma taxa de prevelância de casos de tuberculos reduzida nos últimos anos no Brasil. Porém, esse número ainda é considerado elevado, por se tratar de uma patologia que se for diagnosticada precocemente e completamente tratada, os pacientes com rapidamente se tornam não infecciosos e, eventualmente, curados.

De acordo com Chaves et al. (2017) a tuberculose é uma infecção bacteriana transmitida pela inalação de gotículas da tosse ou espirro de uma pessoa infectada. Ela afeta os pulmões, mas pode se manifestar em todo o corpo, em qualquer sistema do corpo humano, incluindo o sistema nervoso. Além disso, a tuberculose é considerada uma condição potencialmente séria, mas pode ser curada se for tratada com os antibióticos certos.

No entanto, segundo Frieri et al. (2019) os idosos são mais propensos a desenvolver reações adversas a medicamentos de polifarmácia, comorbidades existentes e alterações fisiológicas relacionadas à idade. Isso pode ser explicado pois, os idosos apresentam maior suscetibilidade a doenças infecciosas, principalmente do trato respiratório, e apresentam maior morbimortalidade em relação aos mais jovens. Por isso, a tuberculose ainda impõe altas taxas de mortalidade, especialmente em idosos devido a múltiplas comorbidades e resposta imunológica deficiente.

Portanto, essa pesquisa tem como justificativa o fato de a tuberculose ser uma doença considerada como um problema de saúde pública, por acometer inúmeras mortes ao longo dos anos. Mesmo ela sendo uma patologia que possui cura, a desistência e o segmento adequado do tratamento são fatores responsáveis pelas mortes, principalmente nos idosos. Esse contexto se torna perigoso pois por se tratar de bactérias se o tratamento não for realizado de forma eficaz isso pode causar resistência ao tratamento.

A presente pesquisa teve como objetivo relacionar a atuação do profissional farmacêutico no suporte a idosos com resistência ao tratamento de tuberculose. Com o suporte em descrever sobre a fisiopatologia da tuberculose, apresentar os principais tipos de tratamentos medicamentosos para tuberculose em idosos e relacionar o papel assistência farmacêutica e a resistência ao tratamento em idosos com tuberculose.

\section{Metodologia}

O estudo trata-se de uma revisão de literatura, utilizando o método de pesquisa bibliográfica, pela possibilidade de analisar referências publicadas em estudos já realizados. Com a seleção de informações e conhecimentos prévios acerca da 
temática. Para o levantamento do estudo foram utilizados Livros, Revistas, Diretrizes Sociedades Brasileiras, artigos nos bancos de pesquisas SciELO (Scientific Electronic Library Online) e PubMed (US National Library of Medicine). Para a busca dos artigos, foram utilizados os descritores em português: Assistência farmacêutica, tuberculose; resistência medicamentosa; saúde do idoso.

Foram selecionados 23 estudos, no processo de identificação dessas pesquisas nas bases de dados, foi iniciado com a análise dos títulos e resumos. Sendo excluídos aqueles identificados como não relevantes para a composição deste artigo, também foram excluídos aqueles que apresentaram duplicidade nas bases de dados e os que não estavam disponíveis na íntegra para leitura.

\section{Resultados e Discussão}

\subsection{Assistência farmacêutica no sistema único de saúde (SUS)}

De acordo com Paim (2018) no Brasil foi criado um sistema de saúde pública, onde é oferecido ao povo acesso universal e de forma integrada. Os setores da saúde passaram por inúmeras mudanças, proporcional ao surgimento de novas dificuldades enfrentadas pela população.

Com a criação do Sistema Único de Saúde (SUS), de acordo com Correr et al. (2011) a população brasileira começou a ter acesso à saúde de forma gratuita. O sistema oferece inúmeras assistências de diversas áreas da saúde, nesse contexto está incluso a Assistência Farmacêutica, que de acordo com a Legislação é direito do cidadão. Com a regulamentação da Constituição Federal do Brasil de 1990, a Assistência Farmacêutica passou a fazer parte da atenção integral que deveria ser prestada pelo sistema público de saúde. A Política Nacional de Medicamentos foi concebida num contexto em que era necessário reorganizar a forma de fornecimento de medicamentos aos cidadãos, de acordo com o princípio da descentralização do sistema nacional de saúde.

Para Castro et al. (2014) a assistência Farmacêutica é um conjunto de ações de promoção, proteção e recuperação da saúde coletiva e individual, tendo os medicamentos como elemento essencial para a garantia do acesso e uso racional. Essas ações visam promover a pesquisa, o desenvolvimento e o conhecimento sobre os medicamentos, bem como seus efeitos, utilização e reações adversas, garantia da qualidade do tratamento, monitoramento e avaliação de seu uso, na perspectiva de atingir resultados eficazes e melhoria da qualidade de vida.

Diante disso, entende-se que a Assistência Farmacêutica não está limitada apenas à distribuição de fármacos. Segundo Castro et al. (2015) ela engloba diversas ações e atividades que visam a promoção à saúde e direciona o cuidado diretamente ao paciente. Por contribuir para o uso racional de medicamentos, através de orientações, elaboração de estratégias de acompanhamento da evolução e do uso regular dos medicamentos, principalmente no que diz respeito aos pacientes com tuberculose em tratamento que são submetidos ao uso diária de uma alta quantidade de fármaco e por um longo período de tempo.

\subsection{Fisiopatologia da tuberculose}

Segundo Nogueira et al. (2012) a tuberculose (TB) é uma doença humana causada pelo Mycobacterium tuberculosis. Afeta principalmente os pulmões, sendo a doença pulmonar a apresentação mais comum. Outros sistemas de órgãos comumente afetados incluem o sistema respiratório, o sistema gastrointestinal (GI), o sistema linforreticular, a pele, o sistema nervoso central, o sistema musculoesquelético, o sistema reprodutivo e o fígado.Nos últimos tempos houve um esforço para erradicar a tuberculose. Apesar dos ganhos no controle da tuberculose e do declínio de casos novos e mortalidade, ela ainda é responsável por uma enorme carga de morbimortalidade em todo o mundo. 
Mycobacterium tuberculosis, que causa a tuberculose, é um bacilo álcool-ácido resistente. De acordo com Gagneux (2018) ela faz parte de um grupo de organismos classificados como complexo M. tuberculosis. Outros membros deste grupo são, Mycobacterium africanum, Mycobacterium bovis e Mycobacterium microti. A maioria dos outros organismos micobacterianos são classificados como organismos micobacterianos atípicos ou não tuberculosos.

Segundo Delogu e Fadda (2013) apesar de ser uma infecção pulmonar, a tuberculose é uma doença multissistêmica com manifestação multifacetada. O principal modo de disseminação é através da inalação de gotículas aerossolizadas infectadas. Pois, a capacidade do corpo de limitar ou eliminar efetivamente o agente infeccioso é determinada pelo estado imunológico do indivíduo, fatores genéticos e se se trata de uma exposição primária ou secundária ao organismo. Além disso, M. Tuberculosis possui vários fatores de virulência que dificultam o sistema imunológico na eliminação do organismo de um indivíduo infectado.

De acordo com Gagneux (2018) o primeiro contato do organismo Mycobacterium com um hospedeiro leva a manifestações conhecidas como tuberculose primária. Esta TB primária está geralmente localizada na porção média dos pulmões e é conhecida como o foco de Ghon da TB primária. Na maioria dos indivíduos infectados, o foco Ghon entra em um estado de latência. Este estado é conhecido como tuberculose latente. A tuberculose pulmonar primária é frequentemente assintomática.

Para Bussato et al. (2015) a tuberculose latente é capaz de ser reativada após imunossupressão no hospedeiro. Poucas pessoas podem desenvolver uma doença ativa após a primeira exposição. Esses casos são chamados de tuberculose progressiva primária. A tuberculose progressiva primária é observada em crianças, pessoas desnutridas, pessoas com imunossupressão e indivíduos em uso de esteróides por longo prazo.

Para Nogueira et al. (2012) a maioria das pessoas que desenvolve tuberculose o faz após um longo período de latência (geralmente vários anos após a infecção primária inicial). Isso é conhecido como tuberculose secundária. A tuberculose secundária geralmente ocorre devido à reativação da infecção tuberculosa latente. As lesões da tuberculose secundária estão nos ápices pulmonares. As lesões da tuberculose secundária são semelhantes para reativação e reinfecção quanto à localização.

Segundo Torres (2012) a doença extrapulmonar ocorre em mais de $20 \%$ dos pacientes. O local mais grave é o sistema nervoso central, onde a infecção pode resultar em meningite, que pode ser fatal na maioria dos casos. Outra forma fatal é a infecção da corrente sanguínea por micobactérias, essa forma é chamada de tuberculose disseminada ou militar. A tuberculose extrapulmonar mais comum é a tuberculose linfática. Outras localizações possíveis incluem ossos, articulações, pleura e sistema geniturinário.

\subsubsection{A tuberculose e o idoso}

A idade traz um risco maior de doenças crônicas, como demências, doenças cardíacas, diabetes tipo 2, artrite, câncer e tuberculose. Essas são as principais causas de despesas com doenças, invalidez, mortes e assistência médica no país.

De acordo com Chaves et al. (2017) as características clínicas da tuberculose em idosos podem ser incomuns e podem ser confundidas com doenças relacionadas à idade. As doenças agudas ou crônicas, a desnutrição e as mudanças biológicas associadas ao envelhecimento podem romper as barreiras de proteção, prejudicar os mecanismos de eliminação microbiana e contribuir para a diminuição esperada relacionada à idade nas respostas imunes celulares a micróbios, como o Mycobacterium tuberculosis.

Segundo Oliveira et al. (2013) o diagnóstico de tuberculose pode ser difícil e o tratamento da tuberculose em idosos é desafiador devido ao aumento da incidência de reações adversas a medicamentos. Além disso, os idosos institucionalizados correm um risco especialmente elevado de reativação da tuberculose latente e são suscetíveis a uma nova infecção tuberculosa. 


\subsubsection{Tratamento para tuberculose}

Segundo Dhahri et al. (2014) o tratamento da tuberculose (TB) leva de seis a nove meses e às vezes mais. A tuberculose pode ser curada em quase todos os casos, tomando os medicamentos prescritos pelo médico durante todo o tratamento (pelo menos seis meses). Como todos os medicamentos, os comprimidos anti-tuberculose podem causar efeitos colaterais. $\mathrm{O}$ médico monitora o progresso durante o tratamento para se certificar de que o medicamento está funcionando. Isso geralmente envolve exames de sangue, expectoração ou urina e radiografias de tórax.

Pessoas com infecção tuberculosa latente não apresentam sintomas. No entanto, para Ignatius e Dooley (2019) a bactéria pode se tornar ativa a qualquer momento, portanto, esses pacientes recebem tratamento para evitar que a infecção se transforme em tuberculose. O tratamento e o controle dos casos de infecção tuberculosa latente são fundamentais para a erradicação da doença. Pacientes com tuberculose latente têm menos bactérias da tuberculose em seus corpos do que pacientes com tuberculose ativa, portanto, o tratamento é mais rápido com menos doses. Enquanto a tuberculose ativa requer muitos medicamentos diferentes durante o tratamento, a tuberculose latente requer menos.

De acordo com Busatto et al. (2015) se os pacientes com tuberculose ativa não tomarem todos os medicamentos prescritos ou pararem de tomá-los cedo demais, eles ficarão doentes novamente. Se os pacientes não forem cuidadosos com a dosagem prescrita e as janelas de tempo para tomar o medicamento, a bactéria pode se tornar resistente a determinados medicamentos e, então, requerer tratamento adicional. Pacientes com tuberculose ativa ainda serão contagiosos por algumas semanas em seu tratamento. Os pacientes geralmente são curados após completar todo o regime de medicação exatamente da maneira prescrita pelo médico.

Segundo Junges et al. (2019) os medicamentos podem incluir: isoniazida, rifampicina, pirazinamida e etambutol. O tratamento é oferecido de forma gratuita e está disponível no Sistema Único de Saúde (SUS), sendo implantado juntamente com a realização do regime de Tratamento Diretamente Observado (TDO).

De acordo com Torres (2012) pacientes com tuberculose ativa devem tomar medicamentos por 6 a 9 meses. É essencial concluir todo o tratamento, mesmo que os sintomas desapareçam. Se uma pessoa para de tomar a medicação precocemente, algumas bactérias podem sobreviver e se tornar resistentes aos antibióticos. Nesse caso, a pessoa pode desenvolver tuberculose resistente a medicamentos.

\subsubsection{Resistência ao tratamento em idosos}

De acordo com Frieri et al. (2017) a resistência aos antibióticos é a capacidade da bactéria de não ser suscetível ao antibiótico, o que significa que a bactéria não pode mais ser morta ou parar de crescer para um antibiótico que a mata anteriormente ou interrompe sua multiplicação quando o antibiótico é administrado na dose, via ou administração correta e frequência.

Anzolin et al. (2020) descrevem que os idosos têm maior probabilidade de sofrer efeitos adversos com o tratamento antituberculose do que a população mais jovem e deve-se tomar mais cuidado. Os idosos são mais suscetíveis à resistência de antibióticos devido a alterações fisiológicas e comorbidades. Essa população tem riscos potencialmente maiores de desenvolver doenças relacionadas a essa resistência devido à maior exposição à infecção em ambientes hospitalares.

Segundo Silva et al. (2015) o tratamento da tuberculose é realizado com a utilização de antibióticos, para eliminar as bactérias responsáveis pela doença. A fisiologia do paciente geriátrico já é propensa a desenvolver resistência a determinados tipos de fármacos, por conta de disfunções fisiológicas que estão relacionadas com a idade. Ou seja, por si só o idoso já possui certo nível de intolerância, o que pode ser aumentado com a prescrição e o uso inadequado de antibióticos. 
Por isso, a pesquisa de Silva et al. (2020) conclui ser de grande importância que a equipe de saúde esteja capacitada para enfrentar o contexto que envolve o tratamento da tuberculose. Sendo necessário que esses profissionais desenvolvam método para redução do indice de problemas relacionados a continuidade do tratamento. $\mathrm{O}$ que torna mais emergente, visto que o Programa Nacional de Controle da Tuberculose (PNCT) exige metas a serem alcançadas pelo sistema de saúde.

\subsection{Assistência farmacêutica e a resistência ao tratamento de tuberculose em idosos}

De acordo com Baptista et al. (2013) a resistência aos antibióticos é um dos principais problemas globais que a medicina enfrenta, pois muitos microrganismos estão desenvolvendo resistência aos antibióticos aos quais eram sensíveis. Isso tem um grande impacto nos cuidados de saúde. A resistência a medicamentos, incluindo antibióticos, têm um impacto negativo na qualidade de vida dos pacientes. Um dos problemas que causam resistência aos antibióticos é o uso indevido desses medicamentos.

Porém, para Santos et al. (2012) em muitos casos a resistência ao tratamento da tuberculose ocorre com a falha no segmento do tratamento indicado, muitas vezes o idosos esquece de tomar o fármaco ou acaba tomando na hora errada e até mesmo em muitos casos abandonam o tratamento quando sentem melhorias. Por isso, a atuação da assistência farmacêutica é essencial, principalmente no caso de pacientes idosos, que geralmente realizam o consumo de diversos tipos de fármacos para tratamento de um conjunto de patologias que costumam se desenvolver neste grupo.

Pois, segundo Coutinho et al. (2012) a assistência farmacêutica realizada de forma humanizada e ativa, pode dar suporte na prevenção das falhas da adesão, segmento e continuidade do uso dos medicamentos por idosos, de forma a reduzir o índice de possíveis resistências das bactérias sob os efeitos dos antibióticos prescritos ao tratamento. Além disso, de acordo com Silva et al. (2017) a assistência, se for planejada com atenção e proatividade do profissional farmacêutico pode desenvolver estratégias que busquem desenvolver ações e promoções sobre a importância do segmento adequado do tratamento, o que irá dar suporte nas orientações médicas estabelecidas. Assim como, segundo Nicoletti et al. (2020) o farmacêutico também pode realizar o monitoramento dos resultados terapêuticos, retirada regular dos fármacos do tratamento, além disso a criação de um protocolo farmacêutico específico para os pacientes em tratamento de tuberculose pode auxiliar para rastreamento de possíveis irregularidades no segmento da terapia e os fatores que podem estar associado a dificuldade de adesão eficaz e resistência ao tratamento.

Nesse contexto, a resistência ao tratamento, também é essencial abordar sobre o uso irregular dos medicamentos. Para Silva et al. (2019) quando os medicamentos para tuberculose são tomados, por idosos, de forma inadequada podem causar efeitos colaterais. A maioria dos efeitos colaterais são controláveis, mas alguns podem ser graves. Além disso, os medicamentos podem interagir com outros fármacos que o idoso está tomando. Com isso, é possível compreender que a Assistência Farmacêutica é uma abordagem sistemática, cooperativa e orientada, para obtenção de resultados eficazes da terapia medicamentosa direcionada a qualidade de vida

\section{Considerações Finais}

Com o desenvolvimento da pesquisa foi possível compreender que a tuberculose é uma doença infecciosa que geralmente afeta os pulmões, embora possa afetar qualquer órgão do corpo. Pode se desenvolver quando a bactéria se espalha por meio de gotículas no ar, pode ser fatal, mas em muitos casos é evitável e tratável. Afeta, principalmente idosos, o que torna os riscos associados a ela ainda maiores, visto que se trata de um grupo que possui fragilidades imunológicas e fisiológicas.

Foi possível obter resultados nas literaturas selecionadas, que mostraram que pacientes geriátricos já possuem, naturalmente, resistência a certos tipos de medicamentos, por possuírem inúmeras disfunções biológicas acabam desenvolvendo 
resistência a ação de alguns fármacos, principalmente os antibióticos. O que torna o tratamento da tuberculose, nesse grupo, mais arriscado quanto aos efeitos.

A resistência ao tratamento é reconhecida como uma ameaça ao sistema de saúde. Pois, pode levar a impactos negativos nos resultados dos pacientes, como aumento do tempo de internação hospitalar, declínio funcional, aumento dos gastos com saúde e mortalidade por todas as causas. Também que as práticas inadequadas de prescrição e do uso de antibióticos podem contribuir para a proliferação de bactérias resistentes. Por isso, foi afirmado que a adesão a esse tratamento é muito importante, pois como trata-se de uma terapia contínua e com inúmeros medicamentos, se faz necessário o acompanhamento médico frequente. A eficácia do tratamento vai depender de disciplina e conhecimento do paciente sobre a utilização correta dos fármacos, assim como é essencial que ele entenda os benefícios que o tratamento é feito de forma como é prescrito e orientado pelo médico.

Nesse contexto, que foi associado a participação da assistência farmacêutica, pois um dos principais preceitos da assistência é a prestação direta e responsável de cuidados relacionados com a medicação, tendo como objetivo o alcance dos resultados finitos que melhoram a qualidade de vida do paciente. Além disso, o farmacêutico pode realizar a assistência com o desenvolvimento de estratégias que possam promover a educação e sensibilização dos idosos em relação ao segmento adequado do tratamento. É importante o desenvolvimento de mais pesquisas em relação a esse tema, pois quanto mais os profissionais conseguirem compreender sobre o processo envolvido na resistência que o idoso possui quanto aos medicamentos utilizados no tratamento da tuberculose, mais estratégias podem ser elaboradas para que novos métodos sejam utilizados.

Por isso, é esperado que futuros estudos com utilizem o contexto abordado nessa pesquisa, foquem na atuação do farmacêutico. Abordando a importância da participação direta desse profissional nas intervenções e estratégias desenvolvidas pelas equipes da saúde para redução nas taxas de interrupções no tratamento de tuberculose em idosos.

\section{Agradecimentos}

Agradecemos a todos que contribuíram direta ou indiretamente para que este trabalho se realizasse.

\section{Referências}

Anzolin, A. P., Tavares, L. H., Dalbosco, A. K., Portella, M., Hahn, S., \& Bertol, C. D. (2020). Avaliação das infecções Hospitalares em idosos. Revista Interdisciplinar de Estudos em Saúde, 9(1), 1-10.

Baptista, M. G. D. F. M. (2013). Mecanismos de resistência aos antibióticos (Master's thesis).

Busatto, C., Reis, A. J., Valim, A. R. M., Nunes, L. S., Carneiro, M., \& Possuelo, L. G. (2015). Tuberculose ativa versus Tuberculose Latente: uma revisão de literatura. Journal Infection Control, Rio Grande do Sul, 4(3), 60-4.

Cailleaux-Cezar, M. (2012). Diagnóstico e tratamento da tuberculose latente. Pulmão RJ, 21(1), 41-45.

Osorio-de-Castro, C. G. S., Luiza, V. L., de Castilho, S. R., Oliveira, M. A., \& Jaramillo, N. M. (Eds.). (2014). Assistência farmacêutica: gestão e prática para profissionais da saúde. SciELO-Editora FIOCRUZ.

Chaves, E. C., Carneiro, I. C. D. R. S., Santos, M. I. P. D. O., Sarges, N. D. A., \& Neves, E. O. S. D. (2017). Aspectos epidemiológicos, clínicos e evolutivos da tuberculose em idosos de um hospital universitário em Belém, Pará. Revista Brasileira de Geriatria e Gerontologia, $20,45-55$.

Correr, C. J., Otuki, M. F., \& Soler, O. (2011). Assistência farmacêutica integrada ao processo de cuidado em saúde: gestão clínica do medicamento. Revista Pan-Amazônica de Saúde, 2(3), 9-9.

Costa, R. H. F., da Silva, H. R., de Sousa Matos, R. P., Oliveira, C. J., \& de Brito, M. D. R. M. (2020). Panorama epidemiológico e operacional da tuberculose no estado do piauí: o retrato de uma década. Research, Society and Development, 9(2), e183922088-e183922088.

Coutinho, J., Lopes, J., \& Neto, R. P. (2014). Assistência farmacêutica no programa de tratamento da tuberculose. Revista De Trabalhos Acadêmicos-Campus Niterói.

Silva, L. F., de Carvalho, N. S., de Souza, B. W. L., da Costa Castro, R., Feitosa, A. P. A. R., \& da Silva Martins, V. H. (2020). Fatores de risco e complicações da tuberculose em decorrência do abandono do tratamento. Research, Society and Development, 9(6), e109963501-e109963501. 
Research, Society and Development, v. 10, n. 16, e331101623592, 2021

(CC BY 4.0) | ISSN 2525-3409 | DOI: http://dx.doi.org/10.33448/rsd-v10i16.23592

Delogu, G., Sali, M., \& Fadda, G. (2013). The biology of mycobacterium tuberculosis infection. Mediterranean journal of hematology and infectious diseases, $5(1)$.

Dhahri, B., Aouina, H., Azzabi, S., Baccar, M. A., El Gharbi, L., \& Bouacha, H. (2014). Treatment of tuberculosis. Revue de Pneumologie Clinique, 71(2-3), $122-129$.

Frieri, M., Kumar, K., \& Boutin, A. (2017). Antibiotic resistance. Journal of infection and public health, 10(4), 369-378.

Gagneux, S. (2018). Ecology and evolution of Mycobacterium tuberculosis. Nature Reviews Microbiology, 16(4), 202-213.

Ignatius, E. H., \& Dooley, K. E. (2019). New drugs for the treatment of tuberculosis. Clinics in chest medicine, 40(4), 811-827.

Junges, J. R., Burille, A., \& Tedesco, J. (2019). Tratamento Diretamente Observado da tuberculose: análise crítica da descentralização. Interface-Comunicação, Saúde, Educação, 24, e190160.

Nicoletti, G. P., de Araújo Antunes, A., Gurgel, J. A. R., da Silva Costa, S., \& Brandão, G. H. A. (2020). A importância do profissional farmacêutico no processo de cura da tuberculose. Brazilian Journal of Development, 6(11), 85213-85238.

Nogueira, A. F., Facchinetti, V., Souza, M. V. N. D., \& Vasconcelos, T. R. A. (2012). Tuberculose: uma abordagem geral dos principais aspectos. Rev. Bras. Farm, 93(1), 3-9.

Oliveira, A. A. V. D., Sá, L. D. D., Nogueira, J. D. A., Andrade, S. L. E. D., Palha, P. F., \& Villa, T. C. S. (2013). Diagnóstico da tuberculose em pessoas idosas: barreiras de acesso relacionadas aos serviços de saúde. Revista da Escola de Enfermagem da USP, 47, 145-151.

Paim, J. S. (2018). Sistema Único de Saúde (SUS) aos 30 anos. Ciência \& Saúde Coletiva, 23, 1723-1728.

Pereira, F. A., Zanin, L., \& Flório, F. M. (2020). Avaliação do perfil epidemiológico e indicadores de resultado do Programa de Controle de Tuberculose. Research, Society and Development, 9(8).

dos Santos, D. P., de Souza, D. V., Rodrigues, S., \& Avelar, K. E. S. (2012). Resistência aos fármacos de primeira escolha utilizados no tratamento da tuberculose pulmonar por Mycobacterium tuberculosis. Revista de Saúde, 3(1), 13-25.

Silva, A. C. A., da Cruz, B. O. S., da Costa, E. M., da Silva Carvalho, F., Azevedo, F. H. C., dos Santos, I. A., \& Santos, S. S. S. (2019). Assistência farmacêutica em casos de polifarmácia entre a população idosa. Revista Eletrônica Acervo Saúde, (28), e999-e999.

da Silva, D. B., da Costa, G. S., Rosa, L. F. B., dos Santos Guilherme, M., de Oliveira, S. A., \& de Souza Cavalcanti, R. L. (2017). Assistência farmacêutica a pacientes com tuberculose pulmonar: uma revisão integrativa. Revista Presença, 3(7), 83-106.

Silva, L. M. C. D., Surniche, C. D. A., Sicsú, A. N., Mitano, F., Nogueira, J. D. A., Santos, C. B. D., \& Palha, P. F. (2015). Elaboração e validação semântica de um instrumento de avaliação da transferência do tratamento diretamente observado como política de controle da tuberculose. Revista Panamericana de Salud Pública, 38, 129-135.

Torres Filho, H. M. (2012). Tuberculose. J. bras. med, 53-57. 